\title{
ISLA 2018: NEUE KONZEPTE, JAHRESKONGRESSE IN DREI KONTINENTEN
}

\section{Liebe Kolleginnen und Kollegen, liebe Freunde der ISLA,}

es ist uns eine besondere Ehre, Ihnen heute wieder einmal einVorwort für dieses Heft zu präsentieren und neue Dinge über die ISLA zu berichten.

ISLA heißt ja: ,International Society for Medical Laser Applications", was bedeutet, dass die ISLA medizinische Laserapplikationen beforscht und verbreitet.

Der Name sagt schon, dass dies weit über die (Laser)Akupunktur hinausgeht, die zwar immer noch ein hochspannendes Gebiet darstellt, aber längst nicht mehr das einzige.

Medizinische Laserapplikationen bedeuten regenerative Medizin auf der einen Seite zur Regeneration erkrankten Gewebes, aber auch destruktive Medizin im Bereich der photodynamischen Therapie zur Zerstörung bösartigen Gewebes.

Beide Gebiete haben sich in den letzten Jahren rasant entwickelt: Interstitielle und intraartikuläre Lasertherapie besonders in Kombination mit plättchenreichem Plasma (PRP) haben eine erhebliche Verbesserung der Therapie im Bereich von degenerativen Gelenkerkrankungen und spinalen Syndromen gebracht. Die Kombination mit Fettgewebestammzellen und direkter invasiver Laserknochenmarkstimulation konnte die Ergebnisse weiter verbessern.

Photodynamische Tumortherapien mit dem neuen liposomalen Indozyaningrün unter Anwendung des hoch- dosierten Infrarotlasers haben auch im Gebiet derTumortherapie einen Durchbruch gebracht. Neueste Entwicklungen der infraroten Fluoreszenzspektroskopie von Dr.Weber in Zusammenarbeit mit Dr. Schikora können mittlerweile Tumoren im Frühstadium entdecken, bevor sie ausgebrochen sind, und sind wesentlich sensitiver als MRT-Untersuchungen.

Die neuesten Daten werden von der ISLA auf den eigenen internationalen Kongressen vorgestellt.

Im Juni findet der 14. Internationale ISLA-Kongress in Frankfurt statt, Sie finden die Einladung in diesem Heft. Wer gern auch einmal in die USA reist, vielleicht auch zum „Gambling“, ist herzlich zum ersten US-amerikanischen ISLA-Kongress vom 6.-8.4.18 nach Las Vegas eingeladen. Und wer gern einmal in die pulsierende asiatische Metropole nach Bangkok reisen möchte, ist zum „4. Asian Congress of Medical Laser Applications “ Ende November 2018 eingeladen.

In Las Vegas werden Dr. Weber und Dr. Jennifer Miele von der Tumorklinik in Tijuana in Mexiko von der ,Producer Choice Honors“ in Las Vegas eine spezielle Ehrung für bahnbrechende neue Methoden der Lasertherapie erhalten.

Wir wünschen allen viel Erfolg mit den vielfältigen neuen Methoden und freuen uns, Sie auf unseren Kongressen wiederzusehen.

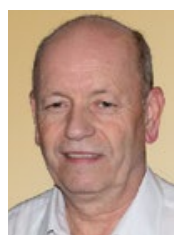

Dr. med. Dipl. Chem. Michael Weber

President for Medical and Clinical Applications der International Society for Medical Laser Applications

(ISLAtranscontinental)

Klinik Neu-Mariahilf, Humboldtallee 10-12,

D-37073 Göttingen, Tel. +49 5273/367 780

E-Mail:info@isla-laser.org,Internet:www.isla-laser.org

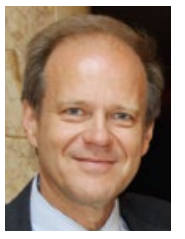

Univ.-Prof. Prof. h.c. DI Dr. techn.

Dr. scient. med. Gerhard Litscher

President for Science and Research der International Society

for Medical Laser Applications (ISLAtranscontinental)

Medizinische Universität Graz, Auenbruggerplatz 29,

A-8036 Graz, Tel: +43 316/385-13907, -83907

E-Mail:gerhard.litscher@medunigraz.at,Internet:http://litscher.info 